\title{
Konvergenz bei Dreiecksformen - interessante geometrische Iterationen
}

\author{
Hans Humenberger · Franz Embacher
}

Eingegangen: 11. April 2017 / Angenommen: 28. Juli 2017 / Online publiziert: 26. Oktober 2017

(C) Der/die Autor(en) 2017. Dieser Artikel ist eine Open-Access-Publikation.

Zusammenfassung In diesem Artikel werden einige bekanntere und einige weniger bekannte geometrische Iterationsprobleme in Zusammenhang mit Dreiecken, In- und Umkreisen behandelt. Die Schwierigkeitsgrade der zugehörigen Begründungen bzw. Beweise variieren dabei beträchtlich: Einerseits könnten einige Probleme durchaus auch in den schulischen Mathematikunterricht Einzug finden, andererseits wird am Schluss des Aufsatzes der Bogen zu fachlich offenen Fragen gespannt. In einem kurzen fachdidaktischen Resumé wird das fachdidaktische Potential des Themas zusammengefasst.

\section{Einleitung}

Viele denken bei Konvergenz zunächst an Zahlenfolgen oder Kontexte aus dem Bereich der Analysis. Es gibt aber auch interessante - und vor allem gut zu veranschaulichende - Konvergenzfragen im Bereich der Elementargeometrie. Die ersten drei der folgenden Probleme sind so elementar, dass sie auch für den Regelunterricht in der Schule in Frage kommen. Das nächste (Problem 4) benötigt den Peripheriewinkelsatz (meist kein Pflichtstoff mehr in der Mittelstufe), ist aber ansonsten strukturell auch so einfach wie die ersten drei. Die weiteren Probleme (5 und 6) werden wohl eher Kursen mit besonders begabten bzw. interessierten Lernenden bzw. Studierenden vorbehalten sein. Besonders hervorgehoben sei schon an dieser Stelle, dass für alle diese Probleme eine Dynamische Geometrie-Software (DGS, z. B. GeoGebra)

\footnotetext{
H. Humenberger $(\bowtie) \cdot$ F. Embacher

Fakultät für Mathematik (und Zentrum für LehrerInnenbildung), Universität Wien,

Oskar-Morgenstern-Platz 1, 1090 Wien, Österreich

E-Mail: hans.humenberger@univie.ac.at

F. Embacher

E-Mail: franz.embacher@univie.ac.at
} 
sehr gute Dienste leisten kann. Man sieht dadurch „viel auf einmal“ in einer eindrucksvollen Art und Weise und wird in den ersten Vermutungen durchaus bestärkt. Die Winkel sind in dieser Arbeit stets als nicht orientierte Winkel aufzufassen.

\section{Elementargeometrische Iterationsprobleme mit dem Inkreis}

\subsection{Die Inkreisberührpunkte bilden das nächste Dreieck}

Problem 1: Gegeben sei ein beliebiges Dreieck $A_{0} B_{0} C_{0}$. Die Berührpunkte seines Inkreises bilden das nächste Dreieck $A_{1} B_{1} C_{1}$. So macht man einige Schritte weiter und kann beobachten, dass die Dreiecke $A_{n} B_{n} C_{n}$, immer gleichseitiger" werden. (Offener: Was kann man bei der Form der Dreiecke $A_{n} B_{n} C_{n}$ beobachten?) Ist das immer so? Lässt sich diese Beobachtung auch beweisen?

In Abb. 1 sind die ersten drei Iterationsschritte mit DGS gezeichnet. Durch Ziehen an den Punkten $A_{0}, B_{0}, C_{0}$ kommt man schnell zur Überzeugung, dass bei diesem Prozess die Form der Dreiecke der „Gleichseitigkeit“ beliebig nahe kommt.

Als zusätzliche Frage (die eigentlich schon ein Hinweis zur Lösung ist) könnte man stellen:

- Was kann über den Winkel $\alpha_{1}$ im Dreieck $A_{1} B_{1} C_{1}$ ausgesagt werden, wenn im Ausgangsdreieck $A_{0} B_{0} C_{0}$ der Winkel $\alpha_{0}$ der kleinste Dreieckswinkel $\left(\alpha_{0} \leq \beta_{0} \leq\right.$ $\left.\gamma_{0}\right)$ ist?

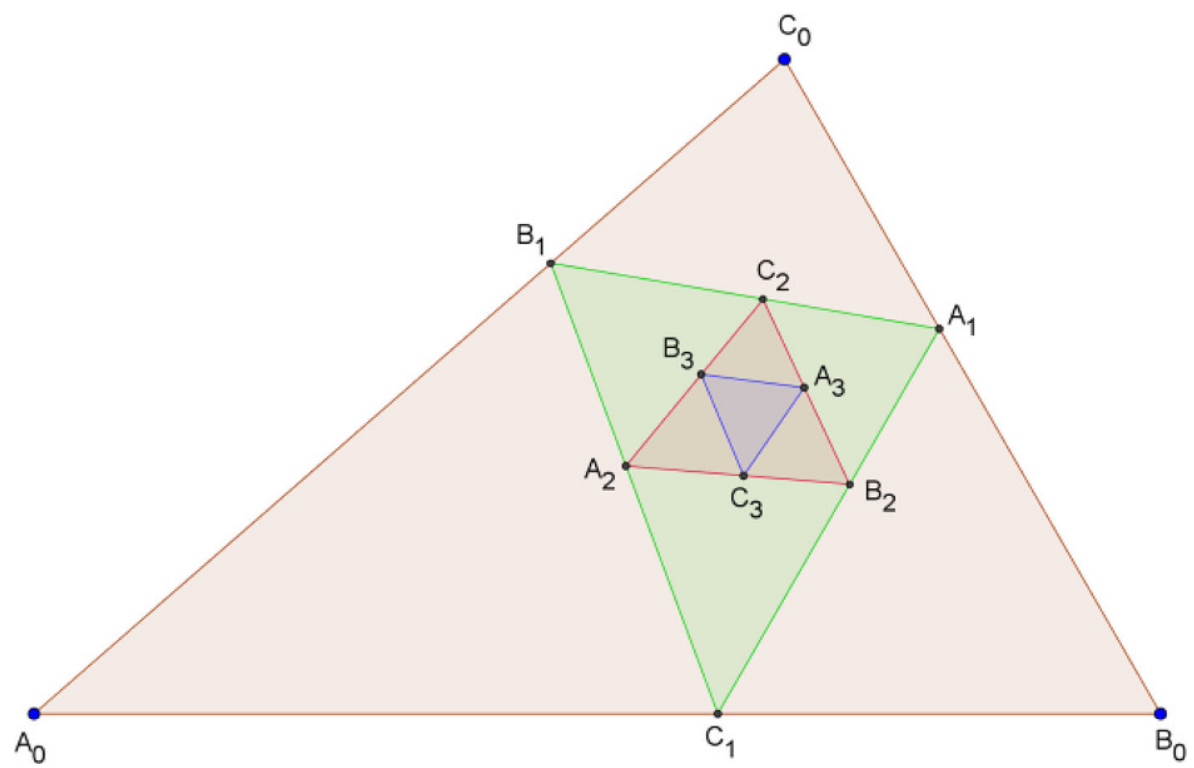

Abb. 1 Drei Schritte im Iterationsprozess „Inkreisberührpunkte“ 


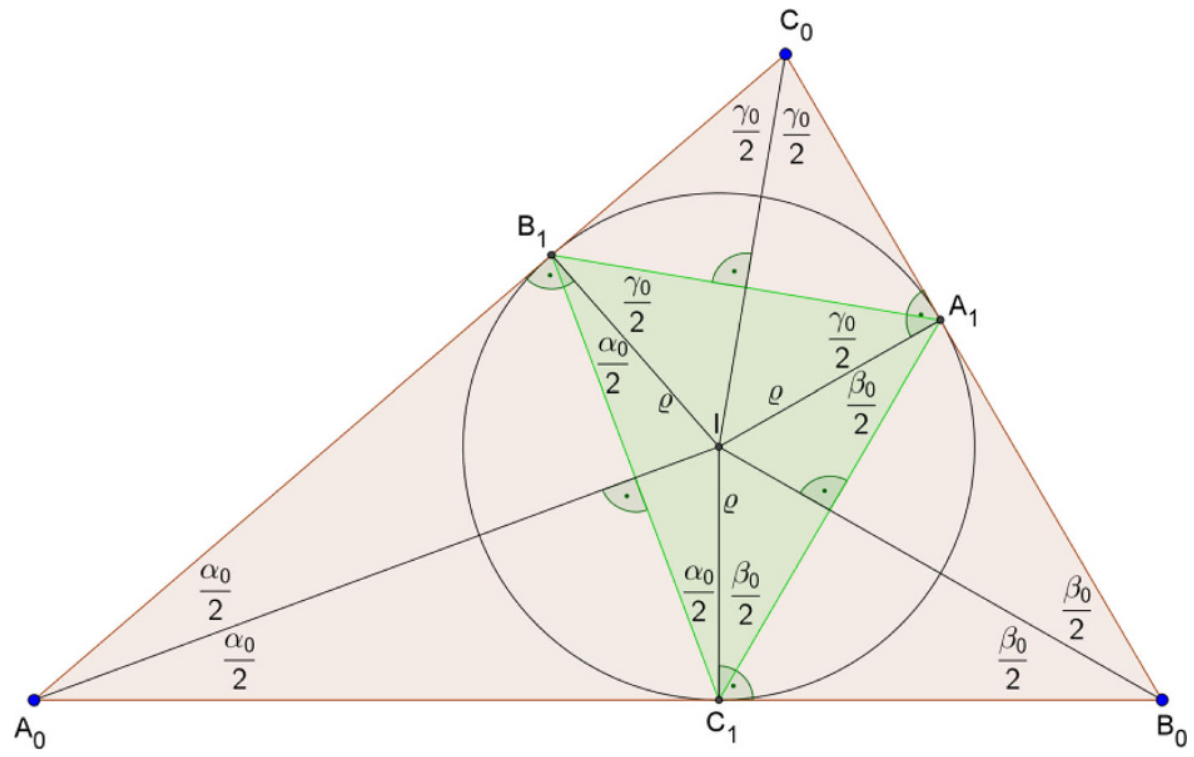

Abb. 2 Zu den Innenwinkeln $\alpha_{1}, \beta_{1}, \gamma_{1}$ bei den Punkten $A_{1}, B_{1}, C_{1}$

Durch diese Zusatzfrage ist es naheliegend, sich mit Winkeln (statt mit Seitenlängen) zu beschäftigen. Eine Winkelmessung mit DGS ergibt, dass es sich bei $\alpha_{1}$ anscheinend um den größten Winkel im Dreieck $A_{1} B_{1} C_{1}$ handelt. Eine Überprüfung auch der anderen Winkel führt auf die Vermutung: $\alpha_{0} \leq \beta_{0} \leq \gamma_{0} \Rightarrow \gamma_{1} \leq \beta_{1} \leq \alpha_{1}$, d. h. bei den Winkeln wird offenbar die Größenreihenfolge ,umgedreht“, dies wird wohl bei jedem Schritt so sein ... Hilft dieses Phänomen bei der Lösung des ursprünglichen Problems (Konvergenz der Form zur Gleichseitigkeit)?

Wie kann man die Beobachtung, dass die Winkel alle dem Wert $60^{\circ}$ zustreben, begründen? Dazu wird man zunächst einmal die einzelnen Winkel $\alpha_{1}, \beta_{1}, \gamma_{1}$ genauer studieren. Die (in Abb. 1 ausgeblendeten) Winkelhalbierenden und der Inkreismittelpunkt spielen nun eine wichtige Rolle (wir beschränken uns auf die ersten beiden Dreiecke $A_{0} B_{0} C_{0}$ und $\left.A_{1} B_{1} C_{1}\right)$.

Weil die Inkreisradien normal auf die Seiten stehen und $A_{0} I \perp B_{1} C_{1}$ gilt (analog $B_{0} I \perp A_{1} C_{1}$ und $C_{0} I \perp A_{1} B_{1}$ ), folgt $\varangle C_{1} B_{1} I=\frac{\alpha_{0}}{2}=\varangle B_{1} C_{1} I$ (analog die anderen Winkel, siehe Abb. 2). Daraus ergibt sich unmittelbar:

$$
\alpha_{1}=\frac{\beta_{0}+\gamma_{0}}{2}, \beta_{1}=\frac{\alpha_{0}+\gamma_{0}}{2}, \gamma_{1}=\frac{\alpha_{0}+\beta_{0}}{2}
$$

D. h. die drei „,neuen“ Winkel sind die paarweise arithmetischen Mittelwerte der „alten“, das ist klarerweise auch bei jedem weiteren Schritt so. Daher ist es schon sehr plausibel, dass sich die Dreieckswinkel einander immer besser annähern, die Unterschiede zwischen ihnen also von Schritt zu Schritt immer besser ,ausgemittelt“ werden. Mit dieser Darstellung der Winkel $\alpha_{1}, \beta_{1}, \gamma_{1}$ erhält man die Implikation $\alpha_{0} \leq \beta_{0} \leq \gamma_{0} \Rightarrow \gamma_{1} \leq \beta_{1} \leq \alpha_{1}$ (analog allgemein: $\alpha_{n} \leq \beta_{n} \leq \gamma_{n} \Rightarrow \gamma_{n+1} \leq$ $\beta_{n+1} \leq \alpha_{n+1}$ ), womit gezeigt ist, dass die Größenreihenfolge bei jedem Schritt 
„umgedreht“ wird. Aus (Gl. 1) folgt sofort eine weitere Darstellung (man bedenke $\left.\beta_{0}+\gamma_{0}=180^{\circ}-\alpha_{0}\right)$ :

$$
\alpha_{1}=90^{\circ}-\frac{\alpha_{0}}{2}, \beta_{1}=90^{\circ}-\frac{\beta_{0}}{2}, \gamma_{1}=90^{\circ}-\frac{\gamma_{0}}{2}
$$

Wie kann man nun strenger beweisen, dass Konvergenz herrscht: $\alpha_{n}, \beta_{n}, \gamma_{n} \rightarrow$ $60^{\circ}$ ? Dafür gibt es mehrere Möglichkeiten:

1. Am einfachsten (und für den Schulunterricht am besten) betrachtet man die Differenzen zum vermuteten Grenzwert $60^{\circ}$ (wir beschränken uns hier auf die $\alpha$ Werte): Die Startdifferenz beträgt $\delta_{0}:=\alpha_{0}-60^{\circ}$, die nächste Differenz beträgt $\delta_{1}=\alpha_{1}-60^{\circ}$, und durch Einsetzen von $\alpha_{1}=90^{\circ}-\frac{\alpha_{0}}{2}$ aus (G1. 2) - man könnte auch (Gl. 1) verwenden - erhält man: $\delta_{1}=30^{\circ}-\frac{\alpha_{0}}{2}=-\frac{1}{2} \delta_{0}$

Dies gilt mit demselben Argument auch für alle weiteren Schritte: Von Schritt zu Schritt halbiert sich die Differenz zu $60^{\circ}$ und wechselt ihr Vorzeichen: $\alpha_{n}-60^{\circ}=$ $\left(-\frac{1}{2}\right)^{n} \cdot\left(\alpha_{0}-60^{\circ}\right) \stackrel{n \rightarrow \infty}{\rightarrow} 0 \Rightarrow \alpha_{n} \rightarrow 60^{\circ}$, d. h. $\lim _{n \rightarrow \infty} \alpha_{n}=60^{\circ}$ (und analog für $\beta_{n}$ und $\left.\gamma_{n}\right)$.

2. Man kann auch mit Intervallschachtelungen argumentieren. Dazu betrachtet man (wieder für den Fall $\alpha_{0} \leq \beta_{0} \leq \gamma_{0}$ ) die jeweils kleinsten Intervalle, in denen die Dreieckswinkel liegen: $I_{0}:=\left[\alpha_{0} ; \gamma_{0}\right]$ mit Länge $\left|I_{0}\right|=\gamma_{0}-\alpha_{0}$. Man erhält $I_{1}=\left[\gamma_{1} ; \alpha_{1}\right]$ mit $I_{1} \subseteq I_{0}$ und Länge $\left|I_{1}\right|=\alpha_{1}-\gamma_{1}=\left|I_{0}\right| / 2$. Dies geht bei jedem Schritt genau so weiter: Das jeweils folgende Intervall ist ganz im vorangegangenen enthalten, und ihre Längen konvergieren gegen 0. Damit ist nach dem Intervallschachtelungsprinzip gezeigt, dass sich die Winkelintervalle $I_{n}$ (in dem sich alle drei Winkel des $n$-ten Dreiecks befinden), ,auf einen Punkt zusammenziehen“, also im Limes alle drei Winkel gleich groß sein müssen, mithin gegen $60^{\circ}$ konvergieren.

3. Eine andere Möglichkeit wäre, mit dem bekannten Satz zu argumentieren, dass monotone und beschränkte Folgen konvergent sind; aufgrund der obigen Betrachtungen ist klar, dass mit $\omega_{k}:=\min \left\{\alpha_{k}, \beta_{k}, \gamma_{k}\right\}(k=0,1,2, \ldots)$ gilt: $\omega_{0} \leq \omega_{1}$ und allgemein $\omega_{n} \leq \omega_{n+1}$, d. h. die Folge $\left(\omega_{k}\right)$ ist monoton wachsend und klarerweise nach oben beschränkt (durch $60^{\circ}$ ), daher muss $\left(\omega_{k}\right)$ konvergieren. Aus den obigen Iterationsformeln (Gl. 1) für die Winkel sieht man aber unmittelbar, dass Konvergenz der Dreieckswinkel nur dann vorliegen kann, wenn alle Winkel gegen den gleichen Wert streben, womit die Konvergenz aller Winkel zu $60^{\circ}$ und damit jene der Dreiecksform zur Gleichseitigkeit gezeigt ist.

Intervallschachtelungen und der eben erwähnte $S a t z$ (monoton und beschränkt $\Rightarrow$ konvergent) existieren zwar vielfach im Schulunterricht, aber nicht in einem hinreichend vertieften und reflektierten Ausmaß, so dass diese Prinzipien zur Verwendung bei Argumentationen wohl nur Studierenden vorbehalten sein werden.

Kann die Konstruktion umgekehrt werden? Es ist hier unmittelbar klar, wie man eindeutig konstruktiv vom Dreieck $A_{1} B_{1} C_{1}$ zurück zum Dreieck $A_{0} B_{0} C_{0}$ kommt: Man zeichnet den Umkreis und die Senkrechten zu den Umkreisradien in den Eckpunkten. Diese Senkrechten schneiden einander in den Punkten $A_{0}, B_{0}, C_{0}$ (vgl. Abb. 2; der Inkreismittelpunkt des Dreiecks $A_{0} B_{0} C_{0}$ ist der Umkreismittelpunkt des Dreiecks $\left.A_{1} B_{1} C_{1}\right)$. 
Eine andere Herangehensweise zu Problem 1 (Matrizen, Grenzwerte von Matrixpotenzen) wird in Ismailescu/Jacobs [5, S. 171f] beschrieben. Dort wird erwähnt, dass der Punkt, dem dieser Prozess zustrebt (d. h. auf den sich die beliebig klein werdenden Dreiecke „zusammenziehen“), Poncelet-Punkt des Dreiecks genannt wird. Auf S. 176 ff wird gezeigt, dass die Gestalt der Dreiecke auch dann der Gleichseitigkeit zustrebt, wenn nicht die Inkreisberührpunkte das jeweils ,nächste“ Dreieck bilden, sondern die Schnittpunkte der Winkelhalbierenden mit ihren Gegenseiten. Der Beweis ist aber nicht elementar (vgl. auch [7]). Weitere Literaturstellen für Problem 1 sind Jones [6, S. 59], Abbott [1, S. 222f] und De Villiers [2].

In De Villiers [2] findet sich auch ein weiteres solches Problem, das dazu passt, obwohl es nichts mit dem Inkreis zu tun hat, sondern mit den Ankreismittelpunkten:

Problem 1a (vgl. De Villiers [2]): Gegeben sei ein beliebiges Dreieck $A_{0} B_{0} C_{0}$. Die drei Ankreismittelpunkte bilden das nächste Dreieck $A_{1} B_{1} C_{1}$. So macht man einige Schritte weiter und kann beobachten, dass die Dreiecke $A_{n} B_{n} C_{n}$ „,immer gleichseitiger“ werden. (Offener: Was kann man bei der Form der Dreiecke $A_{n} B_{n} C_{n}$ beobachten?) Ist das immer so? Lässt sich diese Beobachtung auch beweisen?

Eine strukturell ähnlich einfache Analyse führt auf dieselben Iterationen (Gl. 1) bzw. (Gl. 2) wie bei Problem 1. Das Dreieck der Inkreisberührpunkte (Problem 1) und jenes der Ankreismittelpunkte (Problem 1a) haben nicht nur paarweise gleiche Winkel, sondern auch paarweise parallele Seiten, sie sind also nicht nur ähnlich zueinander, sondern sogar zentrisch ähnlich (vgl. Abb. 3). Das würde man a priori vielleicht nicht unmittelbar erwarten und ist jedenfalls ein interessanter Punkt, der es wert ist hervorgehoben zu werden.

Abb. 3 Zentrische Ähnlichkeit der Dreiecke bei den Problemen 1 und $1 \mathrm{a}$

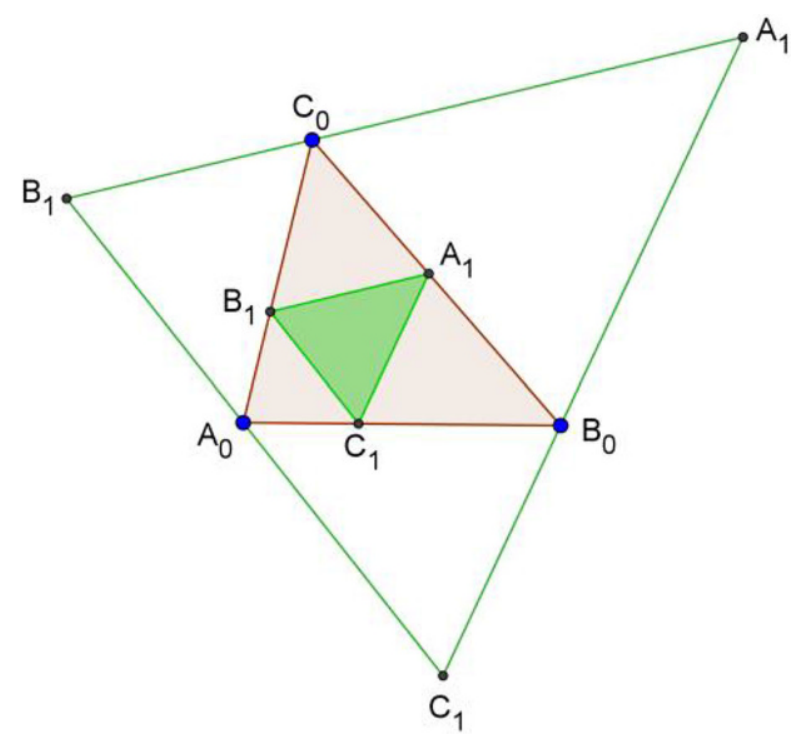


Daher haben wir oben gemeint, dass die Probleme 1 und 1a gut zueinander passen. Es ist auch klar, wie man das zugehörige Ähnlichkeitszentrum konstruiert. Auch hier ist es interessant zu überlegen, wie man die Umkehrung (d.h. vom Dreieck $A_{1} B_{1} C_{1}$ zurück zum Dreieck $A_{0} B_{0} C_{0}$ ) konstruktiv bewältigen würde [Hinweis: Höhen; genauer: $A_{0} B_{0} C_{0}$ ist das Höhenfußpunktdreieck von $\left.A_{1} B_{1} C_{1}\right]$. Ankreise und Ankreismittelpunkte sind im Schulunterricht weniger verbreitet als In- und Umkreise, deshalb wird dieses Problem hier nicht detailliert behandelt, wir begnügen uns mit dem obigen Literaturhinweis.

\subsection{Die Schnittpunkte des Inkreises mit den Winkelhalbierenden (Winkelsymmetralen) bilden das nächste Dreieck}

Das folgende Problem 2 wird in Ismailescu/Jacobs [5, S. 173] beschrieben (und mit Grenzwerten von Matrixpotenzen gelöst). Wir wollen die Lösung wieder möglichst elementar darstellen.

Problem 2: Gegeben sei ein beliebiges Dreieck $A_{0} B_{0} C_{0}$. Die Schnittpunkte seines Inkreises mit den Winkelhalbierenden bilden das nächste Dreieck $A_{1} B_{1} C_{1}$ (vgl. Abb. 4). So macht man einige Schritte weiter und kann beobachten, dass die Dreiecke $A_{n} B_{n} C_{n}$,,immer gleichseitiger" werden. (Offener: Was kann man bei der Form der Dreiecke $A_{n} B_{n} C_{n}$ beobachten?) Ist das immer so? Lässt sich diese Beobachtung auch beweisen?

Aufgrund der konstanten Winkelsumme im Dreieck und der Gleichschenkligkeit der Dreiecke $I A_{1} B_{1}, I B_{1} C_{1}, I C_{1} A_{1}$ ergeben sich für die beiden Teilwinkel bei $A_{1}$ (d. h. Teilwinkel von $\alpha_{1}$ ) die Werte $\frac{\alpha_{0}+\beta_{0}}{4}$ und $\frac{\alpha_{0}+\gamma_{0}}{4}$; damit ergibt sich



Abb. 4 Ein Schritt im Iterationsprozess bei Problem 2 
$\alpha_{1}=\frac{\alpha_{0}+\beta_{0}}{4}+\frac{\alpha_{0}+\gamma_{0}}{4}=\frac{180^{\circ}+\alpha_{0}}{4}=45^{\circ}+\frac{\alpha_{0}}{4}$. Ganz analog erhält man $\beta_{1}=45^{\circ}+\frac{\beta_{0}}{4}$ und $\gamma_{1}=45^{\circ}+\frac{\gamma_{0}}{4}$. Das ist natürlich beim Übergang $n \rightarrow n+1$ genauso, wir erhalten allgemein $\alpha_{n+1}=45^{\circ}+\frac{\alpha_{n}}{4}, \beta_{n+1}=45^{\circ}+\frac{\beta_{n}}{4}, \gamma_{n+1}=45^{\circ}+\frac{\gamma_{n}}{4}$. Man sieht sofort, dass sich die Winkelwerte genau bei $60^{\circ}$ nicht mehr ändern, d.h. $60^{\circ}$ ist der einzig mögliche Grenzwert in diesem Zusammenhang. Die Konvergenz $\lim _{n \rightarrow \infty} \alpha_{n}=60^{\circ}$ lässt sich wieder am einfachsten beweisen, indem man die Differenzen zum vermuteten Grenzwert betrachtet: $\Delta_{n}:=\alpha_{n}-60^{\circ}$. Damit ergibt sich $\Delta_{n+1}=\alpha_{n+1}-60^{\circ}=\frac{\alpha_{n}}{4}-15^{\circ}=\frac{\Delta_{n}}{4}$ und man erkennt, dass die Folge $\left(\Delta_{n}\right)$ eine geometrische Folge mit Faktor $0<q=\frac{1}{4}<1$ und damit eine Nullfolge ist.

Umkehrung: Mit $\alpha_{1}=45^{\circ}+\alpha_{0} / 4$ ist sofort die Eindeutigkeit der Umkehrung dieser Iteration klar. Wie kann man diese konstruktiv möglichst einfach durchführen, d. h. $A_{0} B_{0} C_{0}$ bei gegebenem $A_{1} B_{1} C_{1}$ konstruieren? [Hinweis: Höhengeraden und Umkreis des Dreiecks $A_{1} B_{1} C_{1}$ einzeichnen].

Ein gewisser Nachteil beim Zeichnen der in den Problemen 1 und 2 beschriebenen Dreiecke mit DGS betrifft deren Größe: Sie werden von Schritt zu Schritt kleiner, sogar beliebig klein, so dass man in die Gesamtzeichnung immer tiefer ,hineinzoomen“ muss. Beim Problem 1a werden die Dreiecke immer größer. In Abschn. 3 (Umkreis) treten diese Probleme nicht auf, die Dreiecke bleiben einem festen Kreis eingeschrieben.

\section{Elementargeometrische Iterationsprobleme mit dem Umkreis}

$\mathrm{Zu}$ diesem Thema haben wir in der Literatur nichts gefunden. Wir gehen davon aus, dass die Ergebnisse nicht neu, sondern in der Literatur nur gut versteckt sind. Wir würden uns über Hinweise auf entsprechende Literaturstellen freuen.

\subsection{Umkreis und Mittelsenkrechte (Streckensymmetralen)}

Problem 3: Gegeben sei ein beliebiges Dreieck $A_{0} B_{0} C_{0}$ mit seinem Umkreis $k$. Wir zeichnen die Mittelsenkrechten des Dreiecks $A_{0} B_{0} C_{0}$ und schneiden ${ }^{1}$ diese mit $k$. Dabei ergeben sich die Punkte $A_{1}, B_{1}, C_{1}$ und somit das nächste Dreieck $A_{1} B_{1} C_{1}$. So macht man einige Schritte weiter und kann beobachten, dass die Dreiecke $A_{n} B_{n} C_{n}$,immer gleichseitiger" werden. (Offener: Was kann man bei der Form der Dreiecke $A_{n} B_{n} C_{n}$ beobachten?) Ist das immer so? Lässt sich diese Beobachtung auch beweisen?

Man wird wieder zuerst einige Iterationsschritte mit DGS zeichnen und das geschilderte Phänomen beobachten.

\footnotetext{
1 Die Mittelsenkrechten haben immer zwei Schnittpunkte mit dem Umkreis, man muss dabei festlegen, welche Schnittpunkte man für das nächste Dreieck nimmt: Wir nehmen immer jenen Kreisschnittpunkt, den man erhält, wenn man vom Seitenmittelpunkt Richtung Dreiecksäußeres geht (man könnte auch immer Richtung Dreiecksinneres gehen, dann erhielte man die am Umkreismittelpunkt $U$ punktgespiegelte Version des Dreiecks $A_{1} B_{1} C_{1}$ ).
} 
Abb. 5 a Zentriwinkel 1, b Zentriwinkel 2


Bei der Begründung beschränken wir uns wieder auf den ersten Iterationsschritt $A_{0} B_{0} C_{0} \rightarrow A_{1} B_{1} C_{1}$. Es liegt hier sehr nahe, den Umkreismittelpunkt $U$ ins Spiel zu bringen und die jeweiligen Zentriwinkel der einzelnen Seiten zu betrachten (vgl. Abb. 5).

Wenn die anfänglichen Zentriwinkel zu den Seiten $a_{0}, b_{0}, c_{0}$ mit $\varepsilon_{0}, \mu_{0}, v_{0}$ bezeichnet werden, so sind die neuen Zentriwinkel zu den Seiten $a_{1}, b_{1}, c_{1}$ durch $\varepsilon_{1}=\left(\mu_{0}+v_{0}\right) / 2, \mu_{1}=\left(\varepsilon_{0}+v_{0}\right) / 2, v_{1}=\left(\varepsilon_{0}+\mu_{0}\right) / 2$ gegeben (dies 
stimmt auch, wenn das Dreieck stumpfwinklig ist, der Umkreismittelpunkt also außerhalb des Dreiecks liegt und ein Startzentriwinkel größer als $180^{\circ}$ ist - man überzeuge sich davon durch eine eigene Skizze). Wir können wie bei Problem 1 argumentieren, die „fortgesetzte paarweise Mittelung“ liefert die Begründung für die Konvergenz (intuitiv auf der Plausibilitätsebene oder exakter, nur Zentriwinkel statt Dreieckswinkel). Wir haben hiermit also schon drei Probleme, bei denen einerseits Lernende ohne weitere Voraussetzungen einfach mit DGS die Situation explorieren können und andererseits auch die zugehörige Begründung (zumindest die intuitive mit der Sichtweise der fortwährenden Mittelung bei den Problemen 1 und 3) nicht allzu schwer fallen sollte. Der Hinweis, die Differenzen (oben zu $60^{\circ}$, hier zu $120^{\circ}$ ) genauer zu studieren, kann Anlass für eine formalere Begründung sein.

\section{Bemerkungen:}

- Mit Hilfe des Satzes vom Zentriwinkel (Zentriwinkel sind doppelt so groß wie Peripheriewinkel) ist rasch zu erkennen, dass sich - wie bei Problem 1 - dieselbe Iteration (Gl. 2) bezüglich der Dreieckswinkel ergibt (vgl. Abb. 5a): $\alpha_{1}=90^{\circ}-$ $\alpha_{0} / 2$ etc. Dies haben wir aber bewusst nicht in den Vordergrund gestellt, weil dieser Satz in den Schulen kaum mehr thematisiert wird ${ }^{2}$, die Schüler/innen ihn in der Regel nicht kennen und in selbständiger Arbeit kaum ,entdecken“ werden. Eine Alternative zum Satz vom Zentriwinkel wäre (vgl. Abb. 5b): Man erkennt im Viereck $A_{0} D U E$ (zwei rechte Winkel!) die Beziehung $\alpha_{0}=180^{\circ}-\varepsilon_{1}$ bzw. „einen Schritt weiter“ (d. h. Indices um 1 erhöhen) $\varepsilon_{2}=180^{\circ}-\alpha_{1}$; des Weiteren erhält man $\varepsilon_{0}=360^{\circ}-2 \varepsilon_{1}$ bzw. ,einen Schritt weiter“ $\varepsilon_{1}=360^{\circ}-2 \varepsilon_{2}$. Daraus ergibt sich insgesamt $\alpha_{1}=90^{\circ}-\alpha_{0} / 2$.

- Mit $\varepsilon_{0} / 2=180^{\circ}-\varepsilon_{1}$ (analog für $\mu, v$ ) ist auch klar, dass diese Iteration eindeutig umkehrbar und wie die Umkehrung konstruktiv durchzuführen ist.

\subsection{Umkreis und Winkelhalbierende (Winkelsymmetralen)}

Problem 4: Gegeben sei ein beliebiges Dreieck $A_{0} B_{0} C_{0}$ mit seinem Umkreis $k$. Wir zeichnen nun die Winkelhalbierenden des Dreiecks $A_{0} B_{0} C_{0}$ und schneiden diese mit $k$. Dabei ergeben sich die Punkte $A_{1}, B_{1}, C_{1}$ und somit das nächste Dreieck $A_{1} B_{1} C_{1}$. So macht man einige Schritte weiter und kann beobachten, dass die Dreiecke $A_{n} B_{n} C_{n}$,immer gleichseitiger" werden. (Offener: Was kann man bei der Form der Dreiecke $A_{n} B_{n} C_{n}$ beobachten?) Ist das immer so? Lässt sich diese Beobachtung auch beweisen?

In Abb. 6a sind mit DGS wieder die ersten drei Iterationsschritte gezeichnet.

\footnotetext{
2 In der schulischen Elementargeometrie dominieren in Österreich und Deutschland leider „Berechnungsaufgaben“ (Längen, Flächeninhalte, Volumina), also das Anwenden richtiger Formeln. Die Reichhaltigkeit dieses Gebietes (Sätze, Muster, Begründungen, etc.) geht so verloren, ein Beispiel dafür ist der Peripheriewinkelsatz. Es wäre u. E. an der Zeit diese Entwicklungen zu überdenken und zumindest teilweise rückgängig zu machen, denn an elementargeometrischen Aufgabenstellungen kann das wichtige der Mathematik innewohnende Prinzip des Beweisens exemplarisch und besonders anschaulich thematisiert werden.
} 
Abb. 6 a Drei Schritte im Iterationsprozess ,Winkelhalbierende - Umkreis“, b Zentrische Ähnlichkeit der Dreiecke bei den Problemen 1 und 4

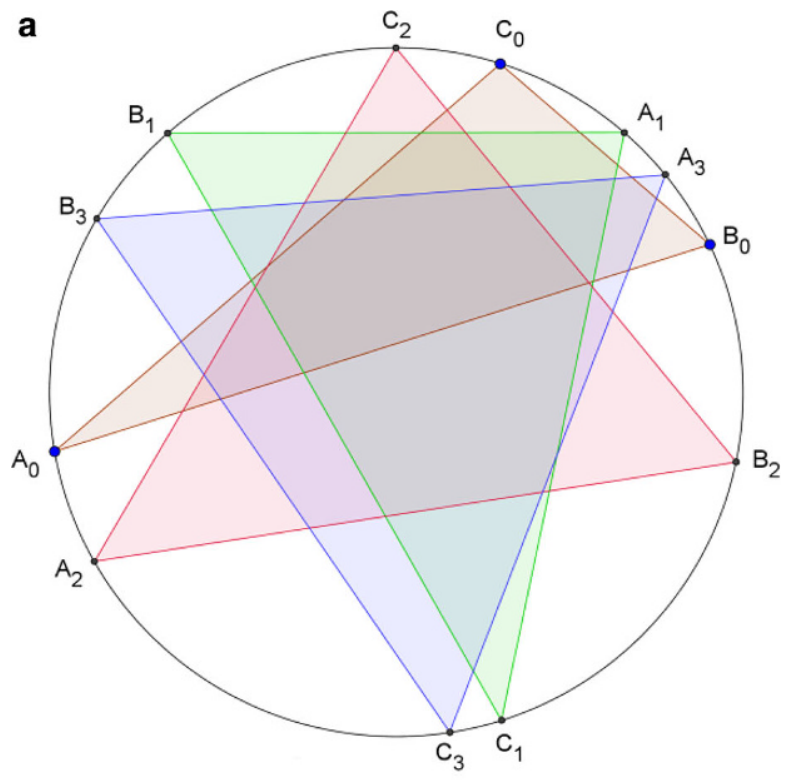

b

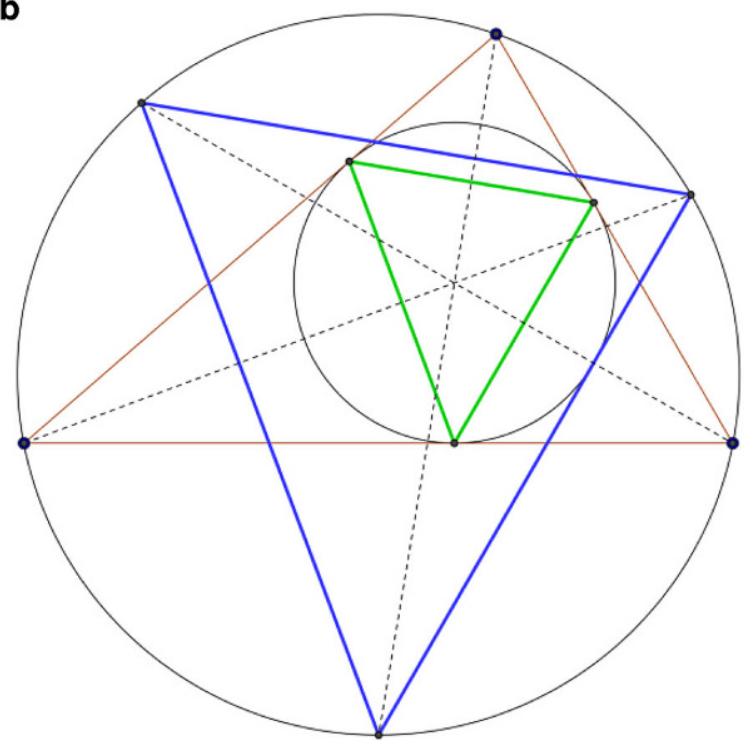

Mit Hilfe des Peripheriewinkelsatzes erkennt man hier: $\alpha_{1}=\frac{\beta_{0}+\gamma_{0}}{2}, \beta_{1}=\frac{\alpha_{0}+\gamma_{0}}{2}$, $\gamma_{1}=\frac{\alpha_{0}+\beta_{0}}{2}$ (vgl. Abb. 7), das sind dieselben Beziehungen wie bei Problem 1 und analog zu jenen bei Problem 3 (dort zunächst Zentriwinkel statt Innenwinkel), d.h. die weiteren Begründungen (intuitiv auf der Plausibilitätsebene oder exakter) verlaufen hier ganz analog zu oben. Die Dreiecke bei den Problemen 1, 3 und 4 sind zueinander ähnlich, mehr noch: wir werden sehen, dass ihre Seiten sogar paarweise zueinander parallel sind. Die Dreiecke der Probleme 1 und 4 (bzw. 3) 
Abb. 7 Die Innenwinkel $\alpha_{1}, \beta_{1}, \gamma_{1}$

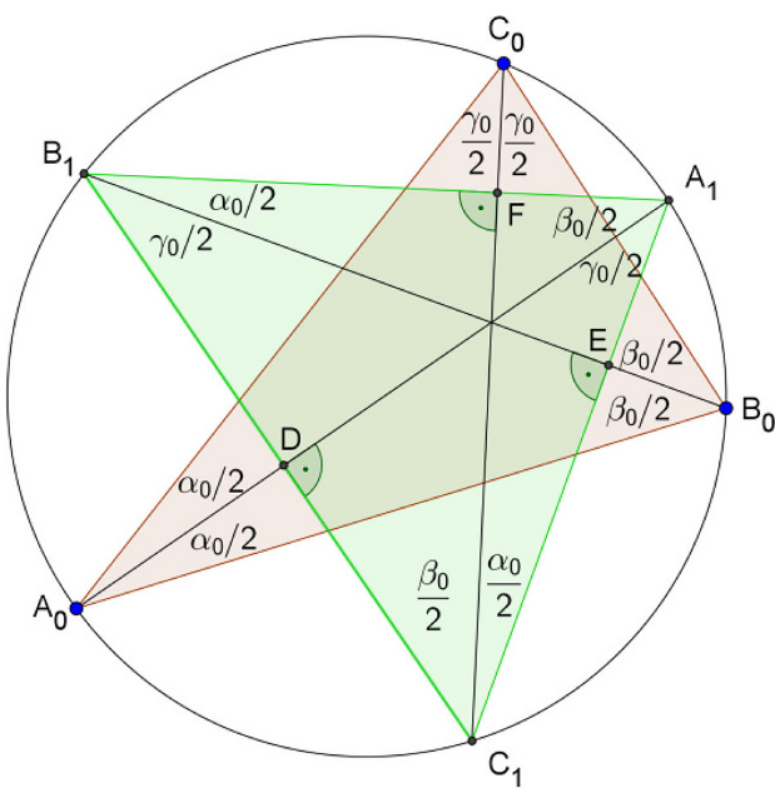

sind sogar zentrisch ähnlich zueinander (vgl. Abb. 6b; das zugehörige leicht zu konstruierende Ähnlichkeitszentrum ist jenes zwischen In- und Umkreis). Im Vergleich zu den Problemen 1 bis 3 kommt bei Problem 4 also der Peripheriewinkelsatz dazu. Aus den Iterationsformeln (auch aus jenen für Problem 3) folgt unmittelbar, dass alle Dreiecke in diesen Folgen (ausgenommen evtl. die Startdreiecke $A_{0} B_{0} C_{0}$ ) spitzwinklig sind!

Bemerkung: Vergleicht man die beiden Dreiecke $A_{1} B_{1} C_{1}$, die sich durch die Winkelhalbierenden bzw. durch die Mittelsenkrechten eines Dreiecks $A_{0} B_{0} C_{0}$ ergeben, so sind das sogar dieselben Dreiecke! Dies mag überraschen und kann zum so genannten Südpolsatz (ein schöner Satz aus der Elementargeometrie) führen:

Die Winkelhalbierende eines Dreiecksinnenwinkels schneidet die Mittelsenkrechte der Gegenseite auf dem Umkreis des Dreiecks.

Dieser Satz ist leicht zu begründen mit dem Peripheriewinkelsatz, vgl. Abb. 8: $S$ sei der Schnittpunkt der Mittelsenkrechten von $A B$ mit dem Umkreis $\Rightarrow|A S|=$ $|S B| \Rightarrow$ die beiden Peripheriewinkel $\varangle A C S$ und $\varangle B C S$ sind gleich groß. Daher ist $C S$ die Winkelhalbierende von $\varangle A C B=\gamma$.

Durch die bekannte Tatsache, dass die Winkelhalbierenden von Dreiecksinnenund -außenwinkel aufeinander normal stehen, ergibt sich mit dem Satz von Thales unmittelbar: Auch die Winkelhalbierenden von Dreiecksaußenwinkeln schneiden die Mittelsenkrechten der Gegenseiten auf dem Umkreis; dies ist dann bildlich gesprochen der „Nordpol“ $N$ des Umkreises, wenn der andere Schnittpunkt als „Südpol“ $S$ bezeichnet wird. Unser Kollege Franz Hofbauer hat deshalb diesen Satz in nahe liegender Weise einmal als „Nordpolsatz“ bezeichnet. 
Abb. 8 ,Südpol“ $S$ und ,Nord-

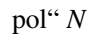

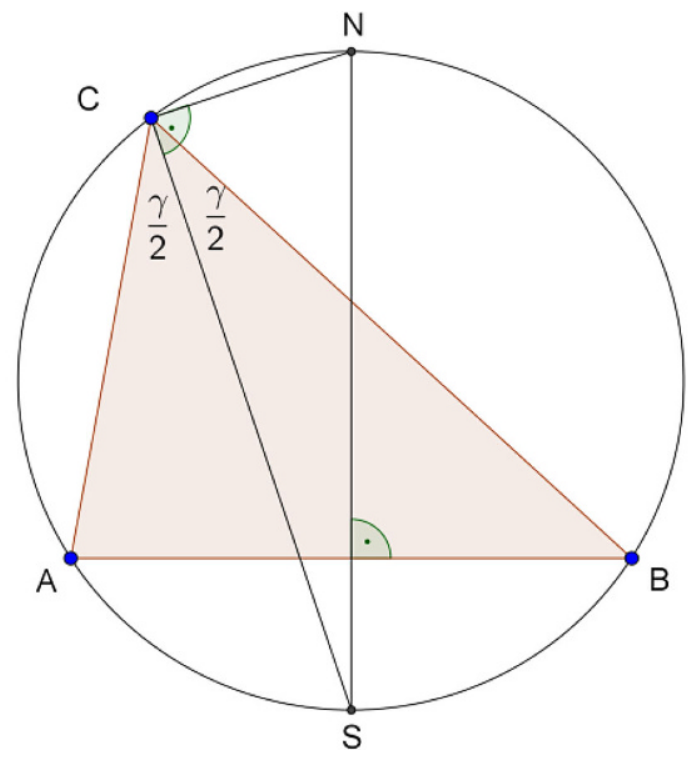

Wenn diese Verbindung zum Südpolsatz hier nicht auffällt, ist dies auch nicht schlimm, denn hier geht es ja um Iterationen und Konvergenzfragen. Aber wenn man entdeckt, dass die Dreiecksfolgen der Probleme 3 und 4 eigentlich dieselben sind, so wird man wohl nach einer Begründung suchen.

\subsection{Umkreis und Höhen}

Problem 5: Gegeben sei ein beliebiges Dreieck $A_{0} B_{0} C_{0}$ mit seinem Umkreis $k$. Wir zeichnen nun die Höhengeraden des Dreiecks $A_{0} B_{0} C_{0}$ und schneiden diese mit $k$. Dabei ergeben sich die Punkte $A_{1}, B_{1}, C_{1}$ und somit das nächste Dreieck $A_{1} B_{1} C_{1}$. So macht man einige Schritte weiter; werden auch hier die Dreiecke $A_{n} B_{n} C_{n}$,immer gleichseitiger"?

DGS-Experimente legen nahe, dass hier wohl eher keine Konvergenz vorliegt (außer im trivialen Fall, wenn man schon mit einem gleichseitigen Dreieck startet). Man kann es entweder bei dieser Erkenntnis belassen oder einen Zusammenhang mit 3.2 (Winkelhalbierende) entdecken. Dieser ist hier gar nicht tief versteckt. Man erkennt in Abb. 7 unmittelbar, dass wegen $\frac{\alpha_{0}+\beta_{0}+\gamma_{0}}{2}=90^{\circ}$ bei den Punkten $D, E, F$ rechte Winkel sind ${ }^{3}$. D. h. wenn man in Abb. 7 im Dreieck $A_{1} B_{1} C_{1}$ die Höhengeraden einzeichnet und mit dem Umkreis schneidet, erhält man das Dreieck $A_{0} B_{0} C_{0}$. Die Prinzipien „Winkelhalbierende einzeichnen und mit dem Umkreis schneiden“ und „Höhengeraden einzeichnen und mit dem Umkreis schneiden“ sind eng miteinander

3 Das beweist nun auch die paarweise Parallelität der Dreiecksseiten bei den Problemen 1 und 4 (zentrische Ähnlichkeit, Abb. 6b), denn sowohl bei Problem 1 als auch bei Problem 4 stehen die Seiten des Dreiecks $A_{1} B_{1} C_{1}$ senkrecht auf die Winkelhalbierenden des ursprünglichen Dreiecks $A_{0} B_{0} C_{0}$. 
verbunden: Sie beschreiben denselben Prozess (dieselbe Iteration), nur in entgegengesetzte Richtungen ${ }^{4}$, wenn jenes Dreieck, dessen Höhengeraden gezeichnet werden, spitzwinklig ist. Im Klartext: Durch das Zeichnen der Höhengeraden können alle Iterationen der Probleme 3 und 4 bis zum jeweiligen Startdreieck $A_{0} B_{0} C_{0}$ rückgängig gemacht werden, denn bei diesen Iterationen ist ja garantiert (siehe oben), dass alle Dreiecke $A_{n} B_{n} C_{n}(n \geq 1)$ spitzwinklig sind. Was dann darüber hinaus passiert, ist noch unklar (siehe unten). DGS-Experimente lassen erkennen: Ausgehend von einem spitzwinkligen Dreieck kann das nächste Dreieck bei der Höheniteration spitz-, recht- oder stumpfwinklig sein, und genauso ist es, wenn man von einem stumpfwinkligen Dreieck ausgeht. Insgesamt geht es da also ,ziemlich durcheinander“. Damit ist schon plausibel, dass beim Problem 5 kaum Konvergenz zu erwarten sein wird.

Weitere systematische Details zur Höheniteration sind im Anhang zu finden.

\subsection{Umkreis und Seitenhalbierende (Schwerlinien)}

Problem 6: Gegeben sei ein beliebiges Dreieck $A_{0} B_{0} C_{0}$ mit seinem Umkreis $k$. Wir zeichnen nun die Seitenhalbierenden des Dreiecks $A_{0} B_{0} C_{0}$ und schneiden diese mit $k$. Dabei ergeben sich die Punkte $A_{1}, B_{1}, C_{1}$ und somit

Abb. 9 Drei Schritte im Iterationsprozess ,Seitenhalbierende Umkreis"

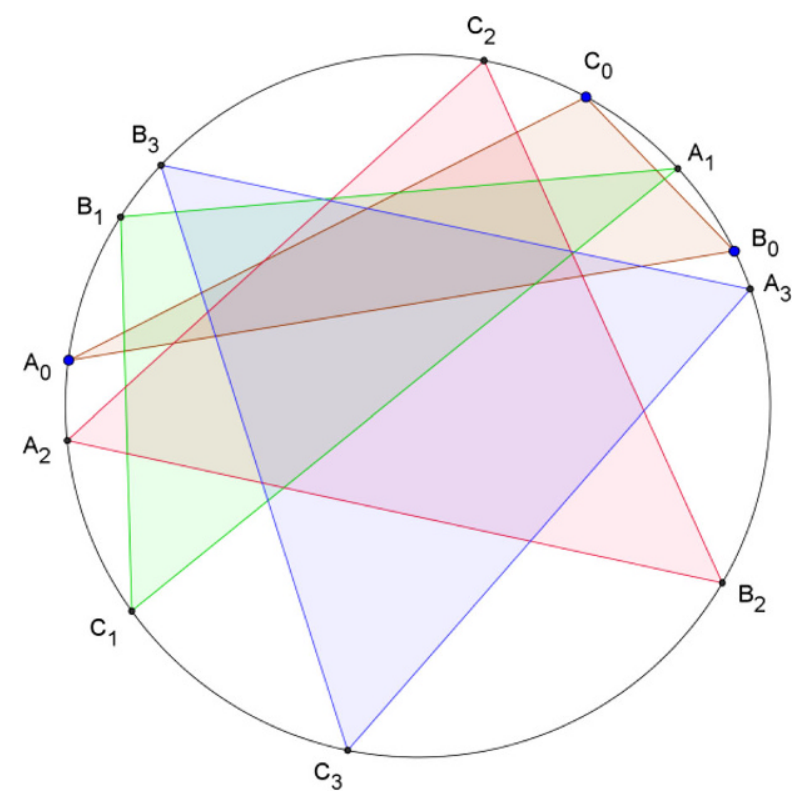

4 Darüber hinaus kann man in Abb. 7 entdecken bzw. erkennen (und bei Bedarf auch begründen): Das Höhenfußpunktdreieck $D E F$ zum Dreieck $A_{1} B_{1} C_{1}$ ist zentrisch ähnlich zum Dreieck $A_{0} B_{0} C_{0}$ mit Streckfaktor 1/2 und dem Höhenschnittpunkt als Ähnlichkeitszentrum. Dies hängt mit zwei bekannten Tatsachen zusammen: „Höhen sind Winkelhalbierende im Höhenfußpunktdreieck“ und „Wenn man den Höhenschnittpunkt an den Seiten spiegelt, dann liegen die Bildpunkte am Umkreis“. 
das nächste Dreieck $A_{1} B_{1} C_{1}$. So macht man einige Schritte weiter und kann beobachten, dass die Dreiecke $A_{n} B_{n} C_{n}$,,immer gleichseitiger" werden. (Offener: Was kann man bei der Form der Dreiecke $A_{n} B_{n} C_{n}$ beobachten?) Ist das immer so? Lässt sich diese Beobachtung auch beweisen?

In Abb. 9 sind wieder drei Iterationsschritte mit DGS gezeichnet.

Wieder scheint es so zu sein, dass die Form der Dreiecke gegen die Gleichseitigkeit konvergiert. Aber wie kann man das beweisen? Die ersten oben genannten Methoden (Folge der „Fehler“ als geometrische Folge erkennen mit $|q|<1$, Intervallschachtelungen) sind hier wenig erfolgsträchtig, aber vielleicht die Idee mit der monotonen Konvergenz? $\mathrm{Zu}$ diesem Zweck müsste man zeigen, dass mit $a_{0} \leq b_{0} \leq c_{0}$ alle drei neuen Seitenlängen $a_{1}, b_{1}, c_{1}$ mindestens so groß wie $a_{0}$ sind. Mit $m_{k}:=\min \left\{a_{k}, b_{k}, c_{k}\right\}(k=0,1,2, \ldots)$ würde dann gelten: $m_{0} \leq m_{1}$ und allgemein $m_{n} \leq m_{n+1}$, d. h. die Folge $\left(m_{k}\right)$ wäre monoton wachsend und klarerweise nach oben beschränkt (trivialerweise durch den Durchmesser des Umkreises bzw. - etwas schärfer - durch die Seitenlänge des zugehörigen eingeschriebenen gleichseitigen Dreiecks), daher müsste $\left(m_{k}\right)$ konvergieren. Da sich beim gleichseitigen Dreieck $^{5}$ die Seitenlängen von einem Schritt zum nächsten nicht mehr ändern, wäre auch klar, dass die Form der Dreiecke der Gleichseitigkeit zustrebt.

Unser Ziel wird also sein zu zeigen: $a_{0} \leq b_{0} \leq c_{0} \Rightarrow a_{1}, b_{1}, c_{1} \geq a_{0}$

Für $c_{1}$ gelingt dies noch relativ einfach (rein elementargeometrisch, vgl. Abb. 10); warum gilt $a_{0} \leq b_{0} \leq c_{0} \Rightarrow c_{1} \geq a_{0}$ ?

Die beiden in Abb. 10 mit $\varepsilon$ bezeichneten Winkel sind Peripheriewinkel über derselben Sehne (nämlich $A_{1} C_{0}$ ) und daher gleich groß. Um zu zeigen, dass $c_{1} \geq a_{0}$ (bzw. $\gamma_{1} \geq \alpha_{0}$ ) ist, genügt es daher zu zeigen, dass $\mu \geq v$ ist, was zum Vergleich von Bogenlängen äquivalent ist: $\left|\widehat{C_{0} B_{1}}\right| \geq\left|\widehat{B_{0} A_{1}}\right|$. Dies gilt aber sicher, weil

Abb. 10 Warum ist $c_{1} \geq a_{0}$ ?

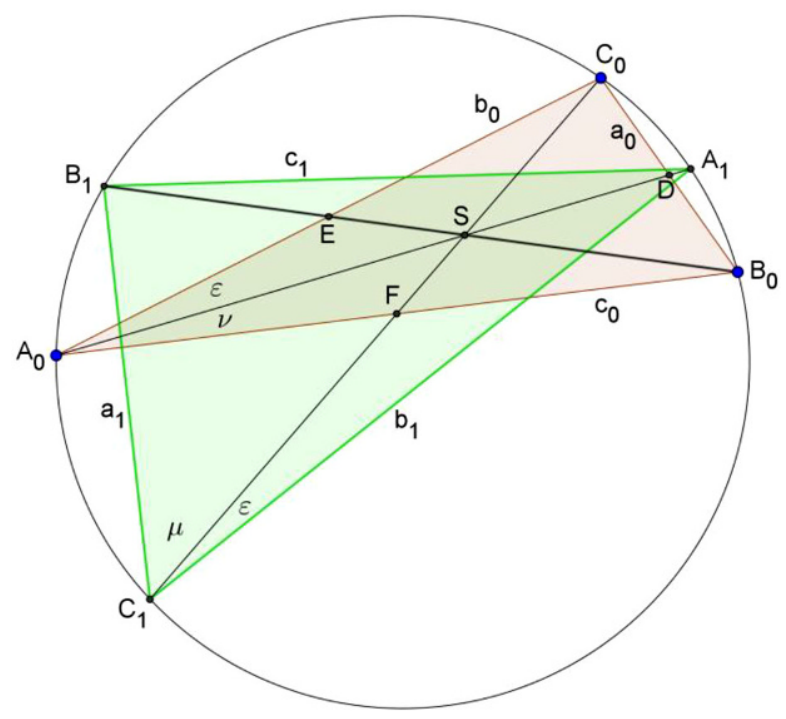

5 Und nur bei diesem (Beweis unten: „Nachtrag“)! 
$\widehat{B_{0} A_{1}}$ höchstens die Hälfte des (kurzen!) Bogens $\widehat{B_{0} C_{0}}$ ausmacht (man beachte: $c_{0} \geq b_{0}$ !), $\widehat{C_{0} B_{1}}$ aber mindestens die Hälfte des längeren Bogens $\widehat{C_{0} A_{0}}$. Damit ist $c_{1} \geq a_{0}$ rein elementargeometrisch gezeigt.

Als nächstes wollen wir zeigen: $a_{0} \leq b_{0} \leq c_{0} \Rightarrow a_{1} \geq a_{0}$

Hier kommen wir (leider) nicht mehr ohne Algebra und Formeln aus. Wir brauchen neben dem Peripheriewinkelsatz zwei weitere Voraussetzungen: Einerseits den sogenannten Sehnensatz (für jeden Punkt innerhalb eines Kreises ist das Produkt der Sehnenabschnitte konstant) und andererseits explizite Formeln für die Längen der Seitenhalbierenden (diese sind leicht durch zweimalige Anwendung des Kosinussatzes zu erhalten):

$$
\begin{aligned}
& s_{a}=\frac{\sqrt{2\left(b_{0}^{2}+c_{0}^{2}\right)-a_{0}^{2}}}{2}, \\
& s_{b}=\frac{\sqrt{2\left(a_{0}^{2}+c_{0}^{2}\right)-b_{0}^{2}}}{2}, \\
& s_{c}=\frac{\sqrt{2\left(a_{0}^{2}+b_{0}^{2}\right)-c_{0}^{2}}}{2}
\end{aligned}
$$

Die Dreiecke $B_{0} C_{0} S$ und $B_{1} C_{1} S$ (vgl. Abb. 10) sind zueinander ähnlich (Peripheriewinkelsatz) und für $\left|E B_{1}\right|$ ergibt sich mit dem Sehnensatz $\left|E B_{1}\right|=\frac{b_{0}^{2} / 4}{s_{b}}=\frac{b_{0}^{2}}{4 s_{b}}$. Daraus erhalten wir $a_{1}=\frac{a_{0}}{\frac{2}{3} s_{c}} \cdot\left(\frac{s_{b}}{3}+\frac{b_{0}^{2}}{4 s_{b}}\right)$. Analog ergibt sich $a_{1}=\frac{a_{0}}{\frac{2}{3} s_{b}}$. $\left(\frac{s_{c}}{3}+\frac{c_{0}^{2}}{4 s_{c}}\right)$.

Wir erhalten so für jede der neuen Seitenlängen $a_{1}, b_{1}, c_{1}$ jeweils zwei Darstellungen (bereits etwas vereinfacht):

$$
\begin{aligned}
& \frac{a_{0} \cdot\left(4 s_{b}^{2}+3 b_{0}^{2}\right)}{8 s_{b} s_{c}}=a_{1}=\frac{a_{0} \cdot\left(4 s_{c}^{2}+3 c_{0}^{2}\right)}{8 s_{b} s_{c}} \\
& \frac{b_{0} \cdot\left(4 s_{a}^{2}+3 a_{0}^{2}\right)}{8 s_{a} s_{c}}=b_{1}=\frac{b_{0} \cdot\left(4 s_{c}^{2}+3 c_{0}^{2}\right)}{8 s_{a} s_{c}} \\
& \frac{c_{0} \cdot\left(4 s_{a}^{2}+3 a_{0}^{2}\right)}{8 s_{a} s_{b}}=c_{1}=\frac{c_{0} \cdot\left(4 s_{b}^{2}+3 b_{0}^{2}\right)}{8 s_{a} s_{b}}
\end{aligned}
$$

Nun zum eigentlichen Beweis von $a_{1} \geq a_{0}$ : Wegen (Gl. 4) ist $a_{1} \geq a_{0}$ äquivalent zu $4 s_{b}^{2}+3 b_{0}^{2} \geq 8 s_{b} s_{c}$. Durch Einsetzen der entsprechenden Terme für $s_{b}, s_{c}$ aus (Gl. 3) erhält man nach wenigen Schritten die äquivalente Ungleichung $b_{0}^{4}+c_{0}^{4} \geq$ $a_{0}^{4}+b_{0}^{2} \cdot c_{0}^{2}$, und diese ist wegen der Voraussetzung $a_{0} \leq b_{0} \leq c_{0}$ immer erfüllt. 
Nun fehlt nur noch der Beweis von $b_{1} \geq a_{0}$, und man ist vielleicht versucht zu glauben, dass der Beweis analog zu jenem von $a_{1} \geq a_{0}$ laufen wird. Das ist aber leider ein (enttäuschender) Irrtum, $b_{1}$ scheint dabei eine gewisse Sonderrolle zu haben. Dieser Sonderrolle kann man ggf. vielleicht durch folgende Aufgabenstellung ein wenig auf die Sprünge helfen:

- Was kann man über den Winkel $\beta_{1}$ (bzw. die Seitenlänge $b_{1}$ ) im Dreieck $A_{1} B_{1} C_{1}$ aussagen, wenn im Ausgangsdreieck $A_{0} B_{0} C_{0}$ der Winkel $\beta_{0}$ der mittlere Dreieckswinkel $\left(\alpha_{0} \leq \beta_{0} \leq \gamma_{0}\right.$, d. h. $\left.a_{0} \leq b_{0} \leq c_{0}\right)$ war?

- Wie ist das für den ursprünglich kleinsten Winkel (kleinste Seitenlänge) bzw. für den ursprünglich größten Winkel (größte Seitenlänge)?

- Probiere zunächst mit DGS (setze es also als Messinstrument ein) und versuche anschließend, die daraus resultierenden Vermutungen zu begründen!

Jetzt sind Winkel und Seitenlängen gleichberechtigt genannt, was dem Problem auch angemessen scheint. Das Resultat der Phase des Explorierens mit DGS wird sein: Es wird vermutlich gelten:

$$
\begin{aligned}
& \alpha_{0} \leq \beta_{0} \leq \gamma_{0}\left(a_{0} \leq b_{0} \leq c_{0}\right) \\
& \Rightarrow b_{1} \text { ist die größte Seitenlänge, d.h. } \\
& a_{1} \leq c_{1} \leq b_{1} \vee c_{1} \leq a_{1} \leq b_{1}
\end{aligned}
$$

Bei den Problemen 1, 3, 4 wurde die Reihenfolge bei jedem Schritt „umgedreht“, d. h. $b_{1}$ war in jedem Fall der ,mittlere“ Wert von $a_{1}, b_{1}, c_{1}$ und die Ordnung der Werte $a_{1}, b_{1}, c_{1}$ stand fest: $a_{1} \geq b_{1} \geq c_{1}$. Jetzt gibt es dazu offenbar gravierende Unterschiede: Es scheint einerseits festzustehen, dass $b_{1}=\max \left\{a_{1}, b_{1}, c_{1}\right\}$ ist. Noch dazu scheint nun die Ordnung zwischen $a_{1}$ und $c_{1}$ zu variieren. Dass hier bei den Seitenhalbierenden ein qualitativ ganz anderes Verhalten besteht als bei den Winkelhalbierenden, mag vielleicht überraschen.

Klar ist aber: Wenn wir (Gl. 7) zeigen können, dann sind wir fertig, dann ist die Konvergenz der Dreiecksform zur Gleichseitigkeit bewiesen, denn dann ist $a_{1}, b_{1}, c_{1} \geq a_{0}$ garantiert.

Wir formulieren (Gl. 7) noch ein wenig um:

$$
\alpha_{0} \leq \beta_{0} \leq \gamma_{0} \quad \text { bzw. } \quad a_{0} \leq b_{0} \leq c_{0} \quad \Rightarrow \quad c_{1} \leq b_{1} \quad \text { und } \quad a_{1} \leq b_{1}
$$

Beweis von $c_{1} \leq b_{1}$ : Wir benutzen Gl. 4,5 und $6, c_{1} \leq b_{1}$ ist äquivalent mit $c_{0} / s_{b} \leq b_{0} / s_{c}$ bzw. mit $4 c_{0}^{2} \cdot s_{c}^{2} \leq 4 b_{0}^{2} \cdot s_{b}^{2}$. Setzt man hier die entsprechenden Terme für $s_{b}, s_{c}$ aus (Gl. 3) ein, so ergibt sich nach wenigen Schritten die wieder äquivalente Beziehung $2 a_{0}^{2} \cdot\left(c_{0}^{2}-b_{0}^{2}\right) \leq\left(c_{0}^{2}+b_{0}^{2}\right) \cdot\left(c_{0}^{2}-b_{0}^{2}\right)$. Diese ist wegen der Voraussetzung $a_{0} \leq b_{0} \leq c_{0}$ immer erfüllt.

Analog erhält man für $a_{1} \leq b_{1}$ die äquivalente Ungleichung $2 c_{0}^{2} \cdot\left(b_{0}^{2}-a_{0}^{2}\right) \geq$ $\left(b_{0}^{2}+a_{0}^{2}\right) \cdot\left(b_{0}^{2}-a_{0}^{2}\right)$, die ebenfalls bei der genannten Voraussetzung immer erfüllt ist.

Bemerkung: Bei der Struktur dieses Beweises scheinen uns zwei Punkte wert, genauer betont zu werden. Einerseits, dass für $c_{1} \geq a_{0}$ Elementargeometrie reicht, 
für $a_{1}, b_{1} \geq a_{0}$ aber nicht, hier sind Formeln und Algebra nötig. Andererseits ist es auch irgendwie erstaunlich, dass die weniger strenge Ungleichung $b_{1} \geq a_{0}$ offenbar schwieriger zu zeigen ist als die strengeren $b_{1} \geq a_{1}, c_{1}$.

Nachtrag: Nur beim gleichseitigen Dreieck ändern sich die Seitenlängen nicht mehr von Schritt zu Schritt (d.h. nur das gleichseitige Dreieck kommt als Grenzwert in Frage). Wir gehen aus von $a_{0} \leq b_{0} \leq c_{0}$ und wissen, dass (Gl. 7) gilt. Im Fall $b_{0}<$ $c_{0}$ erhält man mit denselben Schlussweisen wie oben $c_{1}>a_{0}$ und $a_{1}>a_{0}$, damit kann das Dreieck $A_{1} B_{1} C_{1}$ nicht kongruent zu $A_{0} B_{0} C_{0}$ sein. Im Fall $a_{0}<b_{0}=c_{0}$ kann man auch analog zu oben $b_{1}<b_{0}$ zeigen, woraus wieder die Nichtkongruenz der Dreiecke $A_{0} B_{0} C_{0}$ und $A_{1} B_{1} C_{1}$ folgt. Daher gilt $a_{0}=b_{0}=c_{0}$, das Dreieck $A_{0} B_{0} C_{0}$ ist damit gleichseitig.

Bei der Iteration mit den Winkelhalbierenden, Höhen und Seitenhalbierenden (allgemein: Ecktransversalen) gibt es noch einen interessanten (allgemeineren) Zusammenhang, der aber leider nicht zu einer Vereinfachung des Beweises im Fall der Seitenhalbierenden beiträgt. Es handelt sich um eine Ähnlichkeit spezieller Dreiecke: Wenn man von einem beliebigen inneren Punkt $P$ des Startdreiecks $A_{0} B_{0} C_{0}$ das Iterationsdreieck $A_{1} B_{1} C_{1}$ zeichnet (Schnitt der Ecktransversalen mit dem Umkreis; im Englischen heißen solche Dreiecke ,,circumcevian“, im Deutschen kennen wir dafür keinen Ausdruck) und die Lotfußpunkte $D, E, F$ auf die Seiten des Dreiecks $A_{0} B_{0} C_{0}$ bestimmt, so sind die Dreiecke $A_{1} B_{1} C_{1}$ und $D E F$ immer ähnlich zueinander (vgl. Abb. 11a). Die Begründung ist (auch für sich ganz allein genommen) eine interessante Problemlöseaufgabe. Die Winkel mit gleichem Muster in Abb. 11b deuten den Beweis (mit Peripheriewinkelsatzes und Satz von Thales) schon an.

\section{Weitere ungelöste fachliche Fragen:}

- Mit welchen anderen Ecktransversalen im Dreieck (außer Winkelhalbierende und Seitenhalbierende) ergäbe sich bei der Iteration mit Hilfe des Umkreises ebenfalls Konvergenz der Form zur Gleichseitigkeit?

- Gibt es andere Typen von Ecktransversalen, die bei Iteration (Schneiden mit dem Umkreis) zu anderen interessanten Phänomenen (außer der Konvergenz der Form zur Gleichseitigkeit) führen? Als ein Beispiel kann genannt werden (ohne Beweis): Wenn man jeweils die Verbindungen zum Lemoine-Punkt (oder auch Symmedianenpunkt) nimmt, dann ist $A_{2} B_{2} C_{2}=A_{0} B_{0} C_{0}$, d. h. die Folge der Dreiecke ist ein Zweierzyklus (vgl. [3, S. 77]).

- Die Iteration mit den Seitenhalbierenden ist i. A. nicht eindeutig umkehrbar. Zu gegebenem Bilddreieck $A_{1} B_{1} C_{1}$ (nicht gleichseitig) gibt es jeweils zwei verschiedene Urbilddreiecke (mit gleichem Umkreis wie $A_{1} B_{1} C_{1}$ ), die (mit der Seitenhalbierendeniteration) auf $A_{1} B_{1} C_{1}$ abgebildet werden. Ihre Schwerpunkte sind die Brennpunkte der so genannten "Steiner'schen Inellipse“ des Dreiecks $A_{1} B_{1} C_{1}$. Die zugehörigen Überlegungen liefern spannende Zusammenhänge (z. B. ,isogonal konjugierte Punkte“), die den Rahmen dieser Arbeit deutlich sprengen würden. Kann man diese Zusammenhänge auch elementargeometrisch einsehen? 
Abb. 11 a Situation - Problem, b Lösungshinweis
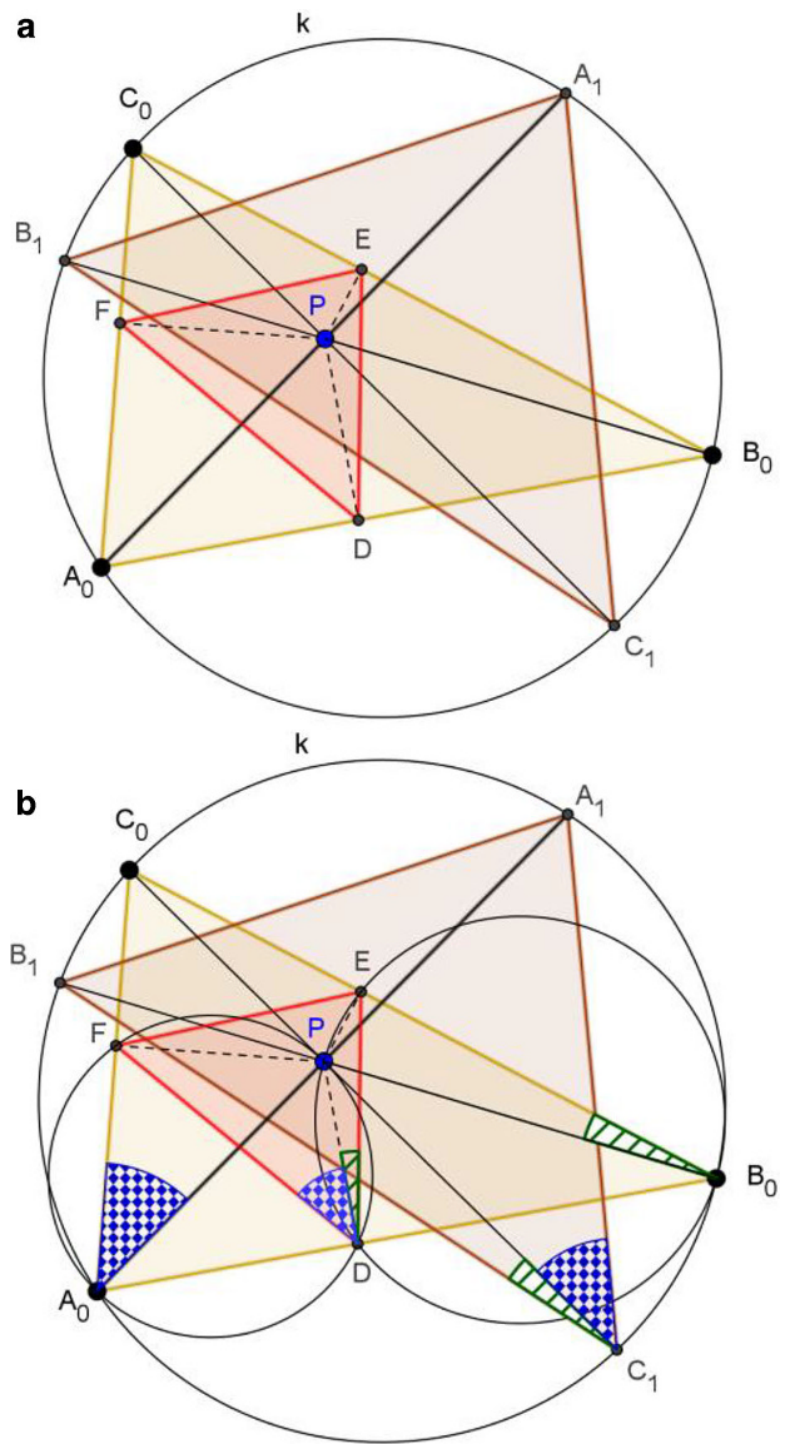

\section{Fachdidaktisches Resumé}

Die Probleme 1-6 sind von ihrem Phänomen her recht einfach. Man kann mit Technologie (Dynamische Geometrie) die jeweilige Situation gut explorieren und so auf Vermutungen bzw. erste Bestätigungen (je nach Aufgabenformulierung) kommen. Hier gestattet es die Dynamik dieser Programme, einen guten Überblick über die Situation zu bekommen. Das sind wertvolle Momente im Prozess des Betreibens von Mathematik. Es sind auch Momente, in denen (wieder einmal) darüber gesprochen werden kann, dass DGS-Experimente natürlich keinen Beweisanspruch (im mathe- 
matischen Sinn) haben können: Erstens kann man ja nie alle möglichen Punkte mit der Dynamik erwischen, so dass nicht einmal das „DASS ES SO IST“ wirklich bewiesen werden kann (man kann darin nur eindrucksvoll bestärkt werden), zweitens kann der bei Beweisen auch sehr wichtige Aspekt „WARUM ES SO IST“ durch DGS-Experimente naturgemäß kaum berührt werden.

Bei den Problemen 1 bis 3 sind die Beweise noch so einfach, dass sie durchaus im Regelunterricht in der Schule stattfinden können. Das Phänomen der fortgesetzten Mittelwertbildung liefert bei den Problemen 1 und 3 schon ein Plausibilitätsargument für die Konvergenz. Ob man dann noch einen mehr formalen Beweis der Konvergenz anstrebt (dafür gibt es mehrere Möglichkeiten, siehe oben), hängt sehr von der Lerngruppe ab. Die Breite an Möglichkeiten zur unterrichtlichen Behandlung ist ein Zeichen für das fachdidaktische Potential der zugehörigen Probleme: Man kann sie auf verschiedenen mathematischen Niveaus behandeln, beginnend vom Regelunterricht der 7. Schulstufe bis zur Universität.

Beim Problem 4 (mit DGS genauso einfach zu realisieren) kommt bei der mathematischen Begründung der Peripheriewinkelsatz dazu. Sollte dieser den Schülerinnen und Schülern nicht bekannt sein, so könnte eine Fragestellung an sie sein: Könnt ihr eine Formel finden, mit der sich $\alpha_{1}$ aus $\beta_{0}$ und $\gamma_{0}$ berechnen lässt? Schreibt euch dazu möglichst viele Winkelwerte (Messen mit DGS) in eine Tabelle. Kommt hier wieder das Prinzip der fortgesetzten Mittelwertbildung zum Tragen? (Die gesuchte Formel ist: $\left.\alpha_{1}=\left(\beta_{0}+\gamma_{0}\right) / 2\right)$ Dadurch können Schülerinnen und Schüler viel entdecken, auch wenn es zu keinem formalen Beweis kommt. Immerhin wäre aber dadurch ein mögliches Tor zum Peripheriewinkelsatz ,,aufgestoßen“.

Die Probleme 5 und 6 werden für eine geometrische Analyse speziellen Kursen mit interessierten Schülerinnen und Schülern bzw. Studierenden vorbehalten bleiben. Aber eine Beschäftigung auf der Ebene der zugehörigen Phänomene (Explorieren mit DGS) ist genau so einfach möglich wie bei den Problemen 1-4.

Insgesamt soll schlussendlich noch einmal betont werden, dass diese Probleme auf verschiedensten Niveaustufen (Unterstufe bis Universität) behandelt werden können - es handelt sich dabei also im Sinne von Hungerbühler [4] um ein ,,skalierbares Thema“ -, wobei Mathematik als Prozess betont wird. Durchaus nahe liegende Verallgemeinerungen und Umkehrprobleme werden dabei vermutlich (auch) zu offenen Fragen und damit zu fachlichem Forschungspotential führen.

Funding Open access funding provided by University of Vienna.

\section{Anhang}

\section{Genaueres zur Höheniteration}

Wir haben oben gesehen, dass im Fall spitzwinkliger Dreiecke die Iteration mit den Winkelhalbierenden genau die „Umkehrung“ der Iteration mit den Höhengeraden ist. Wenn man aber bei einem stumpfwinkligen Dreieck die Höheniteration anwendet, wissen wir über die zugehörige Umkehrung noch nicht Bescheid. Es ist interessant zu überlegen, wie diese Umkehrung konstruktiv geschieht: Angenommen das Dreieck 
Abb. 12 Umkehrung der Höheniteration bei stumpfwinkligen Dreiecken

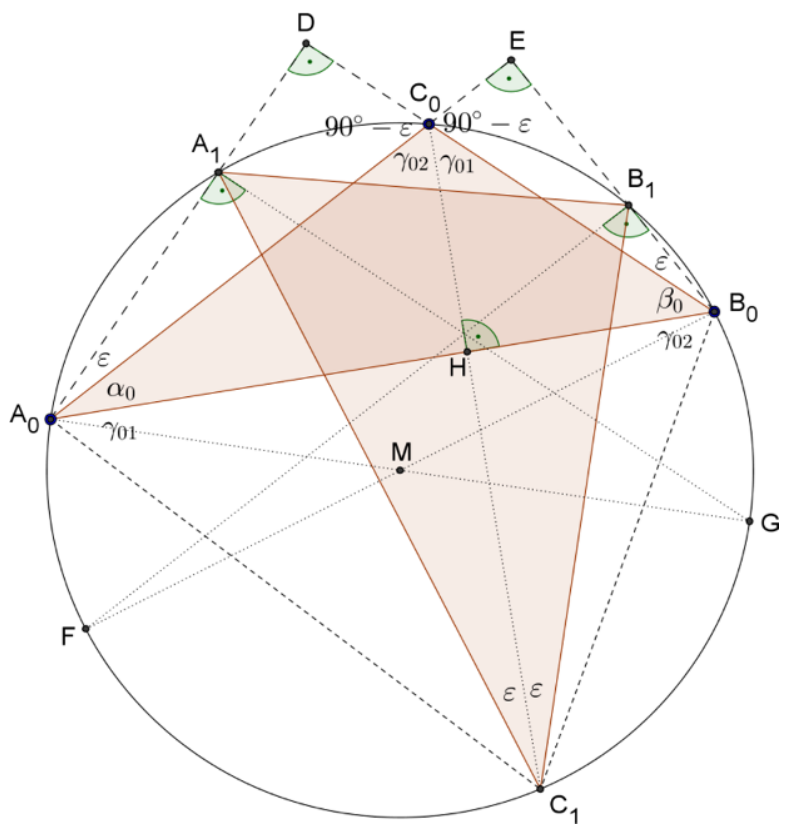

$A_{0} B_{0} C_{0}$ ist stumpfwinklig bei $C_{0}$ und wir kommen durch die Höheniteration zum Dreieck $A_{1} B_{1} C_{1}$. Dann kommt man vom Dreieck $A_{1} B_{1} C_{1}$ konstruktiv wie folgt zurück zum Dreieck $A_{0} B_{0} C_{0}$ :

$C_{0}$ ergibt sich durch Schnitt der Winkelhalbierenden des Innenwinkels bei $C_{1}$ mit dem Umkreis (alternativ: $C_{0}$ ergibt sich durch Schnitt der Mittelsenkrechten von $A_{1} B_{1}$ mit dem Umkreis). Für die anderen beiden Punkte $A_{0}$ und $B_{0}$ schneidet man die Winkelhalbierenden der $A u \beta e n$ winkel in den Punkten $A_{1}$ und $B_{1}$ mit dem Umkreis (vgl. Abb. 12).

Warum ist das so? Eine mögliche Begründung:

Wir verwenden den Peripheriewinkelsatz: $\varepsilon:=\varangle A_{1} A_{0} C_{0}=\varangle A_{1} C_{1} C_{0}$. Bei $C_{0}$ gibt es daher zwei Scheitelwinkel mit dem Maß $90^{\circ}-\varepsilon$. Daher gilt auch $\varangle B_{1} B_{0} C_{0}=$ $\varepsilon=\varangle B_{1} C_{1} C_{0}$ und $C_{0}$ liegt auf der Winkelhalbierenden von $\gamma_{1}$ bei $C_{1}$ bzw. auf der Mittelsenkrechten von $A_{1} B_{1}$.

Im rechtwinkligen Dreieck $A_{0} B_{0} D$ kann man $\alpha_{0}+\varepsilon+\beta_{0}=90^{\circ}$ und im rechtwinkligen Dreieck $H B_{0} C_{0}$ die Beziehung $\gamma_{01}+\beta_{0}=90^{\circ}$ erkennen. Daraus folgt unmittelbar $\alpha_{0}+\varepsilon=\gamma_{01}$. Wegen des Peripheriewinkelsatzes ist $\varangle B_{0} A_{0} C_{1}=\gamma_{01}$ und damit ergeben sich zwei gleiche zugehörige Sehnenlängen $\left|B_{0} A_{1}\right|=\left|B_{0} C_{1}\right|$, d. h. $B_{0}$ liegt auf der Mittelsenkrechten von $A_{1} C_{1}$. Ganz analog folgt, dass $A_{0}$ auf der Mittelsenkrechten von $B_{1} C_{1}$ liegt. Mit dem Südpol- bzw. Nordpolsatz ist obige Behauptung damit bewiesen.

$\mathrm{Zu}$ den Winkelbeziehungen bei der Höheniteration:

- Bei spitzwinkligen Dreiecken $A_{0} B_{0} C_{0}$ mit den Winkeln $\alpha_{0}, \beta_{0}, \gamma_{0}$ liefert die Höheniteration die Winkel $\alpha_{1}=2 \cdot\left(90^{\circ}-\alpha_{0}\right), \beta_{1}=2 \cdot\left(90^{\circ}-\beta_{0}\right), \gamma_{1}=2$. $\left(90^{\circ}-\gamma_{0}\right)$ im Dreieck $A_{1} B_{1} C_{1}$ (dies folgt z. B. aus den Winkeliterationsformeln 
in Problem 4). Dass hier keine Konvergenz zu erwarten ist, ist erstens a priori klar aus der „Umkehrungsbeziehung“ zu den Winkelhalbierenden ${ }^{6}$, zweitens auch aus den eben angegebenen Formeln.

- Bei einem stumpfwinkligen Dreieck $A_{0} B_{0} C_{0}$ mit den Winkeln $\alpha_{0}<90^{\circ}, \beta_{0}<$ $90^{\circ}, \gamma_{0}>90^{\circ}$ liefert die Höheniteration die Winkel $\alpha_{1}=2 \cdot \alpha_{0}, \beta_{1}=2 \cdot \beta_{0}$, $\gamma_{1}=2 \cdot\left(\gamma_{0}-90^{\circ}\right)-$ dies zu begründen ist darüber hinaus eine eigene reizvolle elementargeometrische Aufgabe (vgl. Abb. 13):

Oben haben wir gezeigt: $B_{0} F$ bzw. $A_{0} G$ sind Mittelsenkrechte der Dreiecksseiten $A_{1} C_{1}$ bzw. $B_{1} C_{1} ; B_{1} F$ ist die Winkelhalbierende von $\beta_{1}$ und $A_{1} G$ jene von $\alpha_{1}$. Im rechtwinkligen Dreieck $F B_{0} B_{1}$ erkennt man $\gamma_{01}=90^{\circ}-\frac{\beta_{1}}{2}$, im rechtwinkligen Dreieck $A_{0} H C_{0}$ sieht man $\alpha_{0}+\gamma_{02}=90^{\circ}$. Im Sehnenviereck $C_{1} B_{0} B_{1} A_{1}$ ergänzen Gegenwinkel einander auf $180^{\circ}: \alpha_{0}+\frac{\gamma_{1}}{2}+\frac{\beta_{1}}{2}+90^{\circ}=180^{\circ}$, daraus erhält $\operatorname{man} \gamma_{02}=\frac{\gamma_{1}}{2}+\frac{\beta_{1}}{2}$. Damit ergibt sich einerseits $\alpha_{0}=90^{\circ}-\gamma_{02}=90^{\circ}-\frac{\gamma_{1}+\beta_{1}}{2}=$ $90^{\circ}-\left(90^{\circ}-\frac{\alpha_{1}}{2}\right)^{2}=\frac{\alpha_{1}}{2}$ und andererseits $\gamma_{0}=\gamma_{01}+\gamma_{02}=90^{\circ}+\frac{\gamma_{1}}{2}$. Im rechtwinkligen Dreieck $C_{0} H B_{0}$ sehen wir $\gamma_{01}=90^{\circ}-\beta_{0}$ und damit $\beta_{0}=\frac{\beta_{1}}{2}$. Daraus ergeben sich unmittelbar die behaupteten Iterationsformeln.

Auch dadurch wird man bestärkt, dass Konvergenz kaum vorkommen kann. Interessanterweise gibt es aber doch Fälle, in denen die Höheniteration konvergiert, und zwar auch bei anderen Startdreiecken als dem gleichseitigen. Z. B. bei $\alpha_{0}=30^{\circ}, \beta_{0}=30^{\circ}, \gamma_{0}=120^{\circ}$ ergibt sich $\alpha_{1}=60^{\circ}, \beta_{1}=60^{\circ}, \gamma_{1}=60^{\circ}$, analog erhält man bei $\alpha_{0}=15^{\circ}, \beta_{0}=15^{\circ}, \gamma_{0}=150^{\circ}$ nach zwei Schritten $\alpha_{2}=60^{\circ}, \beta_{2}=60^{\circ}, \gamma_{2}=60^{\circ}$. Andere als diese Art von Startdreiecken

Abb. 13 Winkelbeziehungen bei der Höheniteration

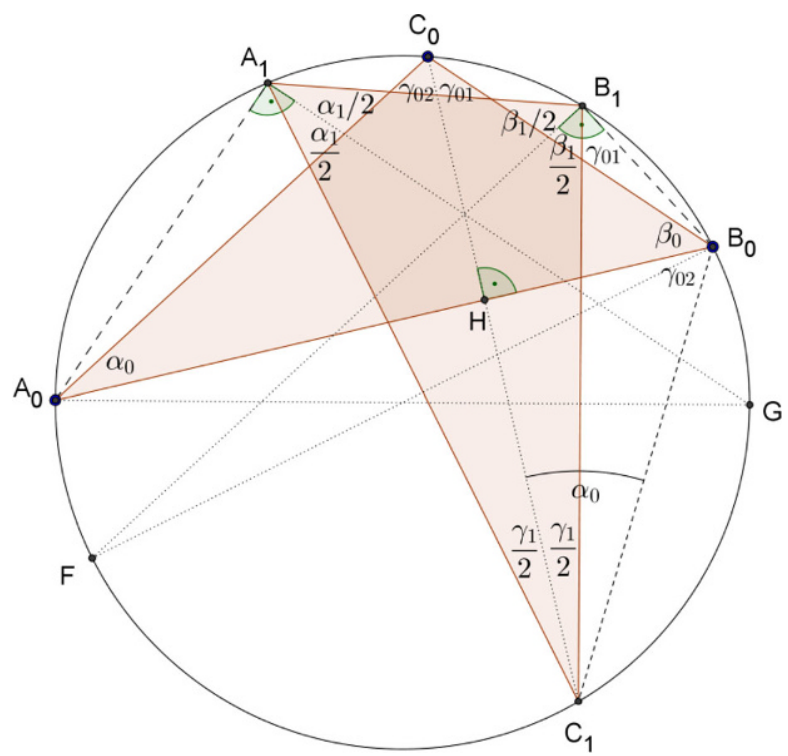

6 Wenn sich beim Prinzip „Winkelhalbierende“ die „Fehler“ (Abweichungen von 60) der Winkel von Schritt zu Schritt halbieren, dann müssen diese sich ja bei der Umkehrung verdoppeln! 
$\left(\alpha_{0}=60^{\circ} / 2^{k}=\beta_{0}, k=0,1,2, \ldots\right)$ kann es für Konvergenz bei der Höheniteration nicht geben.

- Es können bei der Höheniteration auch Zyklen auftreten, ein Beispiel $\left(\alpha_{n}, \beta_{n}, \gamma_{n}\right)_{n \geq 0}$ wäre: $\left(80^{\circ}, 60^{\circ}, 40^{\circ}\right) \rightarrow\left(20^{\circ}, 60^{\circ}, 100^{\circ}\right) \rightarrow\left(40^{\circ}, 120^{\circ}, 20^{\circ}\right) \rightarrow$ $\left(80^{\circ}, 60^{\circ}, 40^{\circ}\right)$

- Ein interessantes Phänomen zur „Umkehrung“ der Höheniteration: Ist ein Dreieck $A B C$ gegeben, dann gibt es genau vier Dreiecke, die ,durch die Höheniteration auf $A B C$ abgebildet werden" und zwar ein spitzwinkliges und drei stumpfwinklige. Diese Dreiecke erhält man aus $A B C$ durch (Gl. 1) Schneiden der inneren Winkelhalbierenden mit dem Umkreis (dieses Dreieck ist das spitzwinklige); für (Gl. 3), (Gl. 4) und (Gl. 5) schneidet man jeweils eine innere Winkelhalbierende mit dem Umkreis - so wie bei (Gl. 1) - und bei den beiden anderen Ecken bringt man die Winkelhalbierenden der Außenwinkel in diesen Punkten zum Schnitt mit dem Umkreis (diese drei Dreiecke sind die stumpfwinkligen). Während die Iterationen bei den Problemen 1 bis 4 eindeutig umkehrbar waren, ist dies bei der Höheniteration also nicht der Fall.

- Bei einem rechtwinkligen Dreieck $A_{0} B_{0} C_{0}$ (rechter Winkel bei $C_{0}$ ) bricht die Höheniteration zusammen, das Dreieck $A_{1} B_{1} C_{1}$ entartet dabei wegen $A_{1}=C_{0}=B_{1}$ zu einem ,Zweieck“.

Im Zusammenhang mit dem rechtwinkligen Dreieck könnte man sich noch überlegen, welche Startdreiecke im Laufe der Höheniteration auf ein rechtwinkliges Dreieck führen, so dass die Iteration abbricht. Für diese Untersuchung muss man zwei Fälle unterscheiden, nämlich spitz- und stumpfwinklige Dreiecke.

- Bei spitzwinkligen Dreiecken ist dies aufgrund der Winkeliterationsformeln $\alpha_{1}=$ $2 \cdot\left(90^{\circ}-\alpha_{0}\right), \beta_{1}=2 \cdot\left(90^{\circ}-\beta_{0}\right), \gamma_{1}=2 \cdot\left(90^{\circ}-\gamma_{0}\right)-$ siehe oben - sofort klar: Es sind spitzwinklige Dreiecke, bei denen einer der Winkel einen der folgenden möglichen Werte hat: $45^{\circ} \leftarrow 67,5^{\circ} \leftarrow 56,25^{\circ} \leftarrow 61,875^{\circ} \leftarrow \ldots$, die Pfeile geben die Richtung der Höheniteration an; in Pfeilrichtung ist die Operation ,die Differenz zu $90^{\circ}$ verdoppeln“ anzuwenden, gegen die Pfeilrichtung die Operation „von $90^{\circ}$ den halben Winkel subtrahieren“.

- Auch bei stumpfwinkligen Dreiecken ist dies aufgrund der Winkeliterationsformeln $\alpha_{1}=2 \cdot \alpha_{0}, \beta_{1}=2 \cdot \beta_{0}, \gamma_{1}=2 \cdot\left(\gamma_{0}-90^{\circ}\right)-$ siehe oben - klar. Es gibt dafür zwei einander nicht ausschließende Fälle: (Gl. 1) stumpfwinklige Dreiecke, bei denen ein spitzer Winkel einen der folgenden möglichen Werte hat: $45^{\circ} \leftarrow$ $22,5^{\circ} \leftarrow 11,25^{\circ} \leftarrow 5,625^{\circ} \leftarrow$..., die Pfeile geben die Richtung der Höheniteration an; in Pfeilrichtung wirkt die Operation ,,verdoppeln“, entgegen die Pfeilrichtung die Operation „halbieren“ (Gl. 3). stumpfwinklige Dreiecke, bei denen der stumpfe Winkel einen der Werte $135^{\circ} \leftarrow 157,5^{\circ} \leftarrow 168,75^{\circ} \leftarrow 174,375^{\circ} \leftarrow \ldots$ hat, in Pfeilrichtung ist die Operation ,,zuerst $90^{\circ}$ abziehen, dann verdoppeln“ anzuwenden, gegen die Pfeilrichtung die Operation ,zu 90 den halben Winkel addieren".

Hiermit liegt eine vollständige Klassifikation vor, in welchen Fällen die Höheniteration konvergent, divergent bzw. abbrechend ist. 
Open Access Dieser Artikel wird unter der Creative Commons Namensnennung 4.0 International Lizenz (http://creativecommons.org/licenses/by/4.0/deed.de) veröffentlicht, welche die Nutzung, Vervielfältigung, Bearbeitung, Verbreitung und Wiedergabe in jeglichem Medium und Format erlaubt, sofern Sie den/die ursprünglichen Autor(en) und die Quelle ordnungsgemäß nennen, einen Link zur Creative Commons Lizenz beifügen und angeben, ob Änderungen vorgenommen wurden.

\section{Literatur}

1. Abbott, S.: Averaging sequences and triangles. Math Gazette 80, 222-224 (1996)

2. De Villiers, M.: Over and over again: two geometric iterations with triangles. Learn Teach Math 16/2014, 40-45 (2014)

3. Honsberger, R.: Episodes in nineteenth and twentieth century Euclidean geometry. The Mathematical Association of America, Washington D.C. (1995)

4. Hungerbühler, N.: Skalierbare Themen im Mathematikunterricht. In: Beiträge zum Mathematikunterricht 2015, S. 26-33. WTM, Münster (2015)

5. Ismailescu, D., Jacobs, J.: On sequences of nested triangles. Period Math Hungarica 53(1-2), 169-184 (2006)

6. Jones, St : Two iteration examples. Math Gaz 74, 58-62 (1990)

7. Trimble, S.Y.: The limiting case of triangles formed by angle bisectors. Math Gaz 80, 554-556 (1996) 